\title{
Power-aware applications for scientific cluster and distributed computing
}

\section{David Abdurachmanov ${ }^{a}$, Peter Elmer ${ }^{* b}$, Giulio Eulisse ${ }^{c}$, Paola Grosso ${ }^{d}$, Curtis Hillegas $^{e}$, Burt Holzman $^{c}$, Ruben L. Janssen ${ }^{d}$, Sander Klous ${ }^{d}$, Robert Knight ${ }^{e}$, Shahzad Muzaffar ${ }^{c}$}

${ }^{a}$ Digital Science and Computing Center, Faculty of Mathematics and Informatics, Vilnius University, Vilnius, Lithuania

${ }^{b}$ Department of Physics, Princeton University, Princeton, New Jersey 08540, USA

${ }^{c}$ Fermilab, Batavia, IL 60510, USA

${ }^{d}$ University of Amsterdam, Kruislaan 904, Amsterdam, The Netherlands

${ }^{e}$ Research Computing, Office of Information Technology, Princeton University, Princeton, New Jersey 08540, USA

E-mail: Peter.Elmerecern.ch

\begin{abstract}
The aggregate power use of computing hardware is an important cost factor in scientific cluster and distributed computing systems. The Worldwide LHC Computing Grid (WLCG) is a major example of such a distributed computing system, used primarily for high throughput computing (HTC) applications. It has a computing capacity and power consumption rivaling that of the largest supercomputers. The computing capacity required from this system is also expected to grow over the next decade. Optimizing the power utilization and cost of such systems is thus of great interest.

A number of trends currently underway will provide new opportunities for power-aware optimizations. We discuss how power-aware software applications and scheduling might be used to reduce power consumption, both as autonomous entities and as part of a (globally) distributed system. As concrete examples of computing centers we provide information on the large HEP-focused Tier-1 at FNAL, and the Tigress High Performance Computing Center at Princeton University, which provides HPC resources in a university context.
\end{abstract}

International Symposium on Grids and Clouds (ISGC) 2014,

23-28 March 2014

Academia Sinica, Taipei, Taiwan

\footnotetext{
* Speaker.
} 


\section{Contents}

1. Introduction 2

2. Computing Model - Current Practice 3

3. Computing Model - Evolution 4

3.1 Transition to multi-core aware software applications 4

3.2 Processor Technology 5

3.3 Data Federations 5

$\begin{array}{lll}\text { 3.4 WLCG as a global power-using computing system } & 6\end{array}$

4. Existing Research on Energy Efficiency 6

5. Example Computer Centers $\quad 7$

5.1 Princeton University Tigress High Performance Computing Center 7

$\begin{array}{lll}5.2 & \text { FNAL Tier } 1 \text { Computing Center } & 7\end{array}$

6. Computing Hardware $\quad 8$

7. Performance-Aware Applications and Scheduling 9

8. Power-Aware Computing $\quad \mathbf{1 0}$

$\begin{array}{lll}8.1 & \text { Simulation results } & 11\end{array}$

9. Conclusions and Future Work 12

\section{Introduction}

Over the past 15 years the use of globally distributed computing resources has been a critical ingredient for many large scientific projects. For example, the Worldwide LHC Computing Grid (WLCG) [1] has been built to serve the needs of the high energy physics (HEP) experiments at the Large Hadron Collider (LHC) [2] at the European Laboratory for Particle Physics (CERN) in Geneva, Switzerland. Numerous scientific results have been produced using the WLCG, including in particular the discovery of the Higgs Boson [3,4], by the CMS [5] and Atlas [6] experiments, which led to the 2013 Nobel Prize in Physics.

The WLCG today brings together computing resources from nearly 160 computer centers in 35 countries, with approximately $350,000 \times 86$ cores of compute power and 200PB of storage, with $10 \mathrm{~Gb}$ network links connecting most centers. Although it is not traditionally considered as a supercomputer, the compute capacity of the WLCG resources is similar to those provided by the 
most powerful supercomputers [7]. Just as importantly, our rough estimates (scaling up from the set of machines currently in use at FNAL) of the power requirements of the WLCG are O(10MW), even before taking into account the Power Usage Effectiveness (PUE) of the participating computer centers. This is again in line with the most powerful supercomputers.

Planning is also underway for a major upgrade, the High Luminosity Large Hadron Collider (HL-LHC) [8], which will run through $\sim 2030$. The data volume increase over the next 15 years will be $\mathrm{O}\left(10^{3}\right)$ and naive extrapolations from today's software indicates that compute needs could increase by factors $\mathrm{O}\left(10^{3}-10^{4}\right)$, significantly faster than usual Moore's Law extrapolations. Significant efforts will be required to reduce that to a level which is possible within funding limits, but it is likely that significant globally contributed compute resources will be continue to be required.

In this paper, we focus in particular on the power aspects of this large distributed computing system. The distributed ownership of the clusters which make up the WLCG implies that the resulting costs are also distributed, however optimizations are in principle possible at all levels. For a given set of hardware, there are three possible goals for throughput-oriented scientific computing applications:

(a) optimizing for maximum performance or throughput (the primary focus today), and/or

(b) optimizing to reduce the total power use for fixed throughput, i.e. the power efficiency (throughput per Watt), and/or

(c) optimizing to reduce total power cost for a given throughput

We will refer back to these three goals in the later sections of this note.

\section{Computing Model - Current Practice}

The construction of the WLCG was greatly facilitated by the convergence, around the year 2000, on Linux and commodity x 86 processors as the standard for HTC scientific clusters like those used in HEP. The resulting homogeneity significantly simplified the use of the compute resources as a "build once, run anywhere" scheduling model was possible for the application software.

The distributed computing and data management models of HEP experiments, however, introduced a simplifying restriction whereby "jobs are sent to data". The computing model of the CMS experiment [9] is a typical example. Datasets are transferred in advance and placed statically in storage (e.g. with tools like PhEDEx [10]) in one or more centers. Each time it is necessary to run an application using data from those datasets as input, the relevant application software is transferred to one of the centers where the required input data can be read via LAN access from sitelocal storage ("data locality"). In order to insure the full utilization of compute resources replicas of data are made manually in multiple sites as required. In the first years of the WLCG many of the computer centers were new and not yet operating at full reliability. The static placement strategy minimized dependencies between centers and allowed for efficient and scalable commissioning of the resources. It had however the disadvantage of requiring significantly more storage than strictly necessary due to the dataset replication. The wide area network (WAN) was also underutilized as a resource, despite being significantly more robust than originally imagined. 
The applications used in HEP are embarrassingly parallel, with no communication required between running instances. Each application simply reads some piece of input data and outputs as a result some processed or reduced version of that data. The applications themselves have a simple, sequential design (no threading). This was consistent with the decades long trend by industry to turn exponential increases in transistor counts over time for the same cost (Moore's Law) into equivalent exponential gains in the performance of sequential software applications like those used in HEP. Around 2005, power density limitations in chips ended this trend, and sequential applications can no longer be made to run exponentially faster on subsequent generations of processors [11]. This is true even if the underlying transistor count continues to increase per Moore's Law. The initial response of industry to these power density limits was the introduction of "multicore" CPUs, with more than one functional processor on a chip. HEP responded to the evolution from single-core to multi-core by simply scheduling additional sequential applications onto the additional cores. In this way net throughput continues to scale roughly with Moore's Law even if individual application performance does not. As a downside, this has greatly increased the number of simultaneous running jobs in the computing systems, increased the total amount of memory needed and placed more stringent requirements on the scalability of job management systems, data access systems, and so forth.

While aggregate power costs and limitations have been important in individual centers, the WLCG system as a whole does not account for or use information about power use or costs operationally, nor do the systems or software used by the WLCG users (i.e. HEP experiments).

\section{Computing Model - Evolution}

The computing model described in Sec. 2 represents the period in which the WLCG was created through the end of the first LHC Run ( 2006-2013). The computing model is now evolving, or under pressure to evolve, for a variety of reasons. In this section we go through the most important changes and argue that, taken together, they enable an operational model where the WLCG can be treated as an integrated, global "power-aware" system.

\subsection{Transition to multi-core aware software applications}

As described earlier, the introduction of multicore processors in 2005 was an epochal change. Power (density) limitations ended the era of Moore's Law scaling for sequential applications, as multi-core processors were introduced. Since the available applications were still sequential (nonthreaded), an "application per core" scheduling model was adopted. As more cores appear in processors, this model led to the use of batch schedulers to schedule ever smaller fractions of the total computing resources (within batch nodes, and within individual CPU dies).

HEP experiments are now in the process of transitioning to multi-core aware (threaded) application frameworks [12-14] which are capable of scheduling work across multiple cores. In the near term, this will resolve some current problems with the "application per core" model, such as ever increasing total memory requirements and scheduler scaling issues with the number of total jobs. In the medium term, however, it represents a significant opportunity for optimization of the processor use, both for performance and for power, at the application level. Although programming multi-threaded applications is more complex than sequential applications, they can potentially 
make choices and coordinate resources for performance and power efficiency better than coarsegrained, incoherent scheduling of independent processes by a batch scheduler. The newer model will be hierarchical: the batch scheduler will schedule a larger resource (e.g. a whole node) and within that the software application will schedule local resources.

For various data management, workflow or bookkeeping reasons, it is also often the case that individual jobs are configured to process a minimum amount of input data (e.g. an entire file or data taken during a fixed amount of time such as a "luminosity section"). This minimum "job size" often implies a minimum wall clock time duration for jobs which can at times be many hours or, in extreme cases, days. Before 2005 increasing processor clock frequencies reduced these minimum job durations over time. In the multicore era, only multi-threaded applications can reduce wall clock times for such jobs. Jobs which complete in a shorter wall clock time, but nonetheless use many processor cores, are useful when power costs vary over the day.

\subsection{Processor Technology}

The same power density limitations that led to multicore CPU's are having additional effects. The performance-per-cost improvement of $40 \%$ or more per year, seen in the 1990's and early 2000's [15], has been replaced by more meager expectations of 20-25\% per year [16], even when taking into account the full use of multicore processors. In the worst case, this corresponds to a factor of 6 growth instead of a factor of $\sim 30$ from Moore's Law over the next decade. The need for larger performance gains drives interest in the use of other processor architectures, including "lightweight" low-power general purpose cores, specialized architectures like GPUs and/or large vector units, etc. These architectures typically have better performance per watt than today's standard general purpose cores, however fully exploiting these newer architectures typically implies significant reengineering of software applications [11,17].

Examples of these alternate architectures are already being deployed today, in the form of coprocessors (GPUs and Intel's Xeon Phi), in computer centers shared with other scientific fields. HEP applications are in general not yet capable of using them, however many preliminary studies have been done [18-25] and a few specific applications are leading the way [26-29]. Several groups have investigated the use of low power mobile processors (e.g. ARM) for HEP applications [3032]. In fact there is some expectation that the power density limitations will lead to System-on-Chip (SoC) [33] architectures mixing special purpose GPUs with lower power general purpose cores. The net effect of this evolution will be significantly more heterogeneity in the computing systems in use, with large variations in performance and power characteristics both within a given center and between centers. In addition significant variation in the efficiency with which applications use any given hardware configuration is likely.

\subsection{Data Federations}

The reliability of all WLCG computer centers has greatly improved from the experience gained during the first LHC run. More sophisticated data management and access models are thus possible. In particular it is now possible to relax the "data locality" requirement. For example, CMS is currently deploying a worldwide data federation [34] using the xrootd data access system to access data remotely across the WAN $[35,36]$. Via the data federation an application running in one center 
can open a file for reading, and the system will find and allow remote reads from a copy of the file wherever it is located in the world. Efficient remote access to data removes compute and storage locality requirements, and allows for many additional job scheduling options for throughput or power efficiency reasons.

\subsection{WLCG as a global power-using computing system}

As described above, the WLCG is evolving. The introduction of multi-threaded applications and the decoupling of locality requirements for storage add significant new flexibility to global job scheduling. At the same time, evolution towards heterogeneous computing hardware and heterogeneous resource provisioning will require more sophisticated approaches to achieve both maximum throughput and power efficient usage. As such, it becomes possible to consider the WLCG not only as a globally distributed computing system, but also as a system with significant power requirements and many opportunities for optimization of the power use.

\section{Existing Research on Energy Efficiency}

Optimization of power costs for large computing centers is of general interest and significant research has been done. Already in 2007 energy proportional computing [37] was identified as an emerging focus area for data centers and large scale infrastructures. The goal in data centers was and is threefold, namely one wants to:

- decrease consumption in order to achieve lower operating costs and reduce environmental impact

- maintain performance by exploiting better energy proportionality of hardware components

- schedule applications in an energy optimal manner.

Reducing the power costs of large scale infrastructures can be accomplished in several manners. [38] and [39] were among the first to propose to exploit the differences in energy pricing in order to place location of computation in a power aware manner. The knowledge of the actual power profiles of the equipment in the data centers and of the network devices connecting them allows to distribute computation and data storage more efficiently, as we have shown in [40].

Exploiting hardware characteristics requires proper models and energy profiles of hardware components. Work in this area abounds: [41] focused on modeling virtual machine's contribution to the consumption of physical nodes; [42,43] proposed statistical power models for GPUs; [44] profiles network equipment. Application scheduling and resource scheduling are emerging now as an integrated approach for large scale distributed systems [45].

In the High Performance Computing (HPC) area, there has been much focus in recent years on Exascale Computing [46,47], including the challenge of limiting the total power consumption of such machines to O(20MW). Exascale computing has some points in common with HTC, in particular regarding the evolution of hardware. However it differs in two important ways. First, synchronization requirements of large parallel HPC applications place more constraints on runtime scheduling choices and use of power saving functionalities (e.g. dynamic voltage and frequency 
scaling, DVFS) than HTC applications, which can be deployed independently in a fine-grained fashion. Second, whereas an HPC installation will be located in a single location with a single hardware architecture, distributed HTC (as in the WLCG) allows for exploitation of geographically heterogeneous power costs and heterogeneous hardware choices.

In this note, we explore the application of some of these ideas to distributed HTC systems, taking into account the specific characteristics of these kinds of applications.

\section{Example Computer Centers}

No full compilation of the energy use of all of the WLCG centers exists at this time. Here we provide some illustrative examples of relevant characteristics for two large computing centers.

\subsection{Princeton University Tigress High Performance Computing Center}

The Tigress High Performance Computing Center at Princeton University is an example of a university research computing facility used for a broad mix of scientific and engineering research purposes including both traditional HPC and batch-oriented high throughput applications. Although not currently integrated into WLCG, it is used as a Tier3 resource by CMS.

The dedicated computer center was built with the intention of replacing the research computing clusters set up by individual departments and research groups in university buildings not designed for that purpose. The center opened in 2011 on the Princeton Forrestal campus. It was designed for up to 5MW utility power into the building and 3MW to the data hall at full build out (currently only $1.8 \mathrm{MW}$ of UPS is provisioned). To supplement the utility power, it has a $2.5 \mathrm{MW}$ emergency diesel generator and a $2.0 \mathrm{MW}$ natural gas generator. The latter is used at times that electricity is expensive, currently 1800+ hours per year. It is designed for a PUE of 1.5 at full build out, and currently varies 1.4-1.7. Typical power use today is $1.2 \mathrm{MW}$.

Power costs range from $\sim \$ 0.08$ to $\$ 0.80$ / $\mathrm{kW}$ hour in New Jersey, with an average of $\$ 0.09$ $\$ 0.10 / \mathrm{kW}$-hour. Princeton pays on a minute by minute basis. The co-generation plant on campus includes predictive modeling to know when to turn on and off. The natural gas generator leverages this capability to know when to turn on and off. While not all electricity markets have this kind of dynamic pricing, in places where it exists it provides for work scheduling choices to reduce power cost.

\subsection{FNAL Tier 1 Computing Center}

Fermi National Accelerator Laboratory (FNAL), in Batavia, IL (USA) provides Tier-1 computing center resources as part of the CMS experiment. Fig. 1 shows the average CPU utilization efficiency over the past year for the set of nodes comprising the WLCG Tier1 computing center. The average CPU efficiency varies over time and in practice is often less than $100 \%$. Fig. 2 shows the utilization by job type for both the Tier1 and for a dedicated analysis cluster. Two things are visible. First, utilization of all available job slots is high, but not always 100\%. At times some slots will be idle. Second, the origin of the variation in CPU utilization efficiency is clearer: jobs can be classified into two rough categories: "production" jobs with high CPU efficiency and "analysis" jobs with lower efficiency. The analysis jobs typically need to read more data (relative to computation) and are waiting on input data. (The change in job mix can in part be attributed to the fact that 


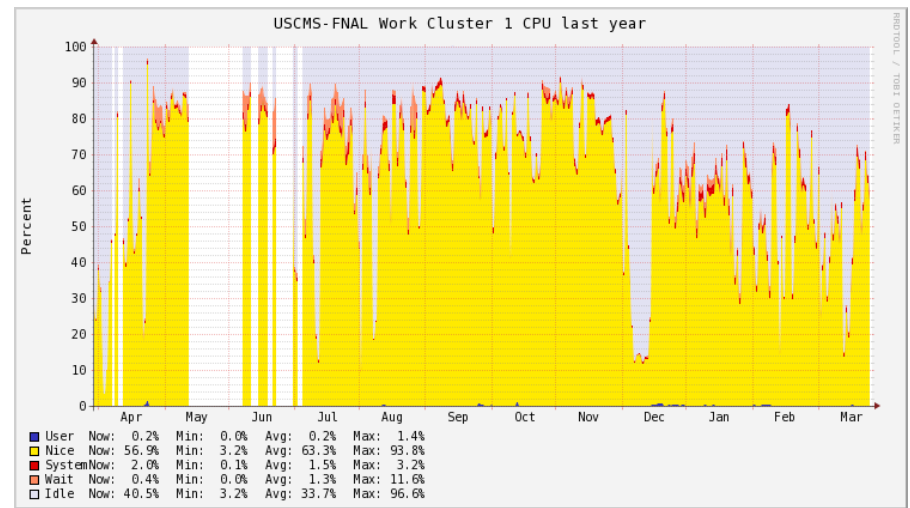

Figure 1: Average CPU utilization of nodes at FNAL Tier1 site.

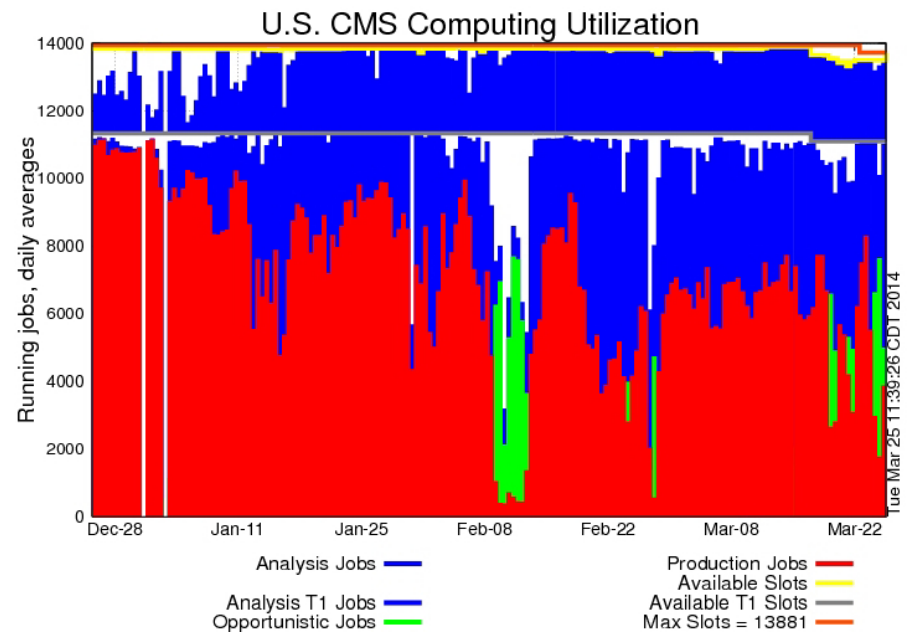

Figure 2: Job slot (core) utilization, by job type, for FNAL Tier1 site. The lower plot is for the Tier1 grid cluster, the upper plot is for the LPC analysis cluster.

early 2014 corresponds to the midpoint of a 2 year shutdown of the LHC for upgrades, and thus a reduced need for "production" jobs and a higher fraction of analysis jobs.) The type characteristics of jobs are often known or can be measured, as 100's or 1000's of jobs of a given type are usually submitted together. Again, work scheduling choices can reduce power cost.

\section{Computing Hardware}

The power consumption of individual hardware components, in particular processors, is one important ingredient for the optimization of overall power use and cost. Most WLCG sites have multiple purchases of computing nodes made over the past 4-5 years, with some reporting nodes in use for up to 8 years after purchase. Different generations of nodes having wildly different performance per unit power characteristics. (Up to a factor of 20 in the case of 8 years of purchases.) In addition, the future introduction of either low-power cores or co-processor cards (GPUs, Xeon Phi), coupled with varying application efficiency in using them, will introduce further heterogeneity. 


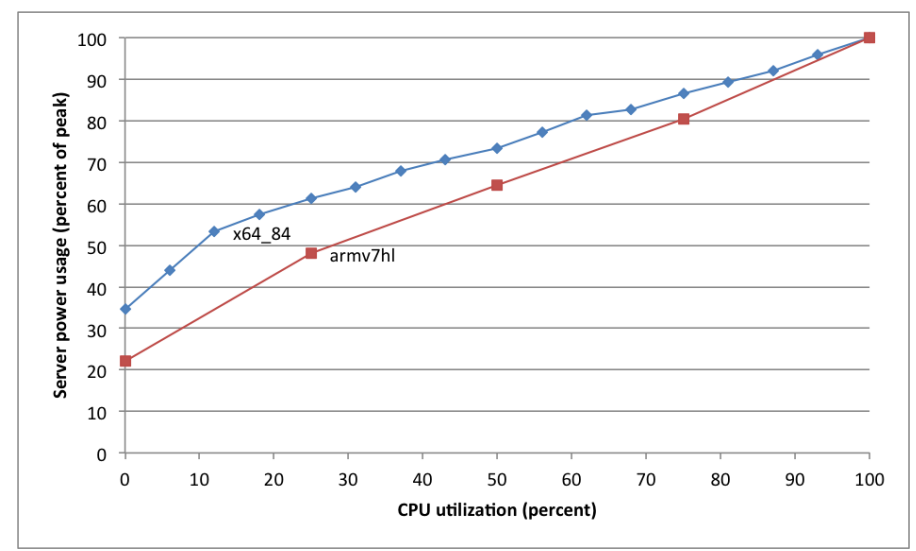

Figure 3: ARMv7 (Exynos 5410, A15, performance governor, 1.6Ghz) Efficiency, x86-64 (Intel(R) Xeon(R) CPU E5-2670 0 @2.60GHz) Efficiency

When jobs are not $100 \%$ efficient an additional important aspect is the power proportionality of the systems. Fig. 3 shows one such comparison, between a standard x 86 server and (for illustration) a simple development board for a low-power processor. In cases where CPU utilization is less than $100 \%$ due to job inefficiency or due to empty job slots (idle cores), these curves illustrate the varying cost of different machines. Also in this case, the introduction of co-processors will change significantly these curves, and their importance will grow as application efficiency in using the co-processors may vary significantly. For hardware combinations that are not fully powerproportional (i.e. consuming zero power for zero throughput), the most power-efficient working point is of course $100 \%$ utilization, but this is not always achievable.

Note that the non-zero idle power contains contributions not only from the (idle) processor, but also from the motherboard, power supply, internal disks and the bulk memory. HTC clusters are often built to "worst case" application requirements and typically extra memory and/or local disk capacity is added because of small purchase-time cost. As power use and cost become more important, characterization of power proportionality curves at purchase time will also help quantify eventual operational costs from such choices.

\section{Performance-Aware Applications and Scheduling}

Before considering the power-related aspects of applications and scheduling, we first consider additional emerging opportunities from performance-aware applications and scheduling, i.e. goal (a) in Sec. 1.

An application's performance can be characterized by its throughput, CPU efficiency, memory use, etc. Today's simple, sequential applications are however typically not designed to measure anything about their performance because they were not designed with any significant run-time adaption to change their behavior. At best they make such measurements, but purely for reporting purposes. All "adaption" is usually left to external entities such as the workflow management system or even to the user/programmer to adapt in the next version of the program.

As described in Sec. 3.1 and 3.2 application designs are evolving to use multiple cores and specialized processing capabilities such as GPUs, explicitly taking on work- and resource-scheduling 
responsibilities that sequential applications lacked. These designs in principle can evolve to measure performance and adapt the amount and type of work they schedule within the application to maximize throughput. Typically they do this today in a basic fashion at job startup, e.g. by noting the number of cores and/or GPUs available and configuring the job to use them. Similar optimizations are possible for memory and local disk use for caches. A logical next step would be for the job to adapt itself as it runs based on performance measurements, e.g. by starting additional threads, scheduling larger chunks of input data, etc. This kind of fine-grained performance optimization cannot be done by a batch scheduler, but only by the software application itself.

Performance measurements from the application itself, especially in terms of "average throughput" achieved, can also be used for more effective job scheduling. (We use throughput, meaning average output per unit time, and not absolute output, as the latter will vary depending on particular job configurations.) Already today, sequential application performance will vary from (general purpose) core to core depending on processor type (CPU, cache memory, etc.). How it varies will also depend on the specifics of the particular application type. Note that it should be possible for each application type to measure "throughput" on its own terms. Rather than use the absolute value of that quantity for scheduling purposes, suitably normalized (across processor type) averages can be calculated and the marginal advantage of any particular scheduling choice could in principle be calculated and comparisons made between different application types. This becomes even more interesting as hardware becomes heterogeneous and application designs permit running on multiple hardware configurations (e.g. code which runs via OpenCL on both CPUs and GPUs). In this way, scheduling choices can be made to maximize the overall throughput of the heterogeneous system with a mix of application types.

\section{Power-Aware Computing}

Once optimizations to achieve maximum throughput have been made, a logical next step would optimization of power use or cost for a given throughput, i.e. goals (b) and (c) from Sec. 1. We consider a number of cases.

In the simple case where there is insufficient work in the queue (i.e. job slots are empty, as is seen from time to time in Fig. 2), two possibilities exist. The simplest solution is that new jobs can be scheduled on the most power efficient hardware possible. For general purpose CPUs, the power efficiency can simply be determined via one-time hardware characterization and used for all subsequent scheduling choices. For more complex heterogeneous mixes or varying CPU utilization efficiencies, power-aware applications might be needed to provide both throughput and power use characterization of each application type. Measuring the power requires both hardware and operating system support (e.g. via interfaces such as IPMI). A more complex solution would be aggregation (via scheduling or virtual machine migration) of all jobs on the most power efficient nodes and (temporary) powering down unused nodes if their idle power is non-negligible.

In the more common case where a sufficient queue of work exists, but the applications have a mix of processor utilization efficiencies, two possibilities exist. Processor utilization efficiencies can be less than $100 \%$ for multiple reasons, including latencies due to input and output (I/O) of data, insufficient parallel computation (for multi-threaded or codes which use coprocessors) and/or insufficient memory. As in the previous section one can still define a throughput metric for each 
application type on its own terms, and similarly a throughput per power metric, and use these to do job scheduling. Note that this naturally encompasses situations where a particular application cannot use a coprocessor card such as a GPU, but is able to use the host CPUs on the same machine. The gain in terms of relative throughput for each application type for scheduling choices as well as idle power is treated quantitatively.

In the presence of process inefficiency and when sufficient memory is available, one strategy today to increase the aggregate throughput is to overschedule the compute resource with multiple jobs. Some sites in fact do this, however it is limited by the available memory and in cases where $\mathrm{I} / \mathrm{O}$ is limited by filesystem access rather than latencies it may be counterproductive. In addition, overscheduling of emerging architectures (larger numbers of lower power cores, coprocessor cards) is more complex than general purpose cores.

Thus the two methods for exploiting a mix of application efficiencies both aim to reduce power cost, but exploiting temporal or geographical price variations in power cost. As described in the Tigress example in Sec. 5.1, power costs can vary dynamically in some centers and some component of this variation is predictable (e.g. day/night or peak/off-peak use variations). The temporal power-aware scheduling choice within a single center would be to schedule the most processor efficient jobs (with the highest power needs) during the periods of lowest power cost, and those with lower processor utilization efficiency during the periods of higher cost. Net throughput would in principle remain the same on average. Similarly, a geographical power-aware scheduling choice is available on distributed computing systems like the WLCG which takes advantage of the fact that the phase of temporal variation and average local power costs vary from center to center.

\subsection{Simulation results}

A simple simulation [48] allowed us to quantify the relation between possible performance improvements and energy savings in the WLCG under different scheduling policies. We focused on seven Tier2 sites, for which we knew the processor types present and the job history from information in the CMS Dashboard [49]. Fig. 4 shows the scheduling scheme we simulated; we relied on two sets, a processor set and an available job set, to couple jobs and processor with each other. The processor set distinguishes available nodes in terms of their performance. The job set relies on information from actual WLCG job logs to classify incoming jobs as low and high CPU efficient based on the author previous running history.

Once a processor becomes available the scheduling algorithm will find the best job in the job pool for the specific processor. For our experiments we used three different types of algorithms: (1) FIFO scheduling selects the first initiated job in the queue is executed first; (2) Energy efficient scheduling aims to schedule CPU intensive jobs on relatively energy efficient processors. This results in lower total energy consumption as CPU intensive jobs benefit more than the lesser CPU intensive jobs of the better energy profile of the processor; and (3) CPU performance scheduling schedules jobs to processors with a performance that is proportional to the estimated relative CPU efficiency of the job. Fig. 5 shows that both the performance and energy scheduling significantly reduce energy consumption in comparison to FIFO scheduling; the average improvements over the seven sites are respectively $6.74 \%$ and $7.07 \%$. Similarly Fig. 6 shows an improvement in the performance of the two efficiency-oriented algorithms over the basic scheduler. The average improvement in this case is $3.44 \%$ for the CPU scheduling and $2.95 \%$ for the energy scheduling. 


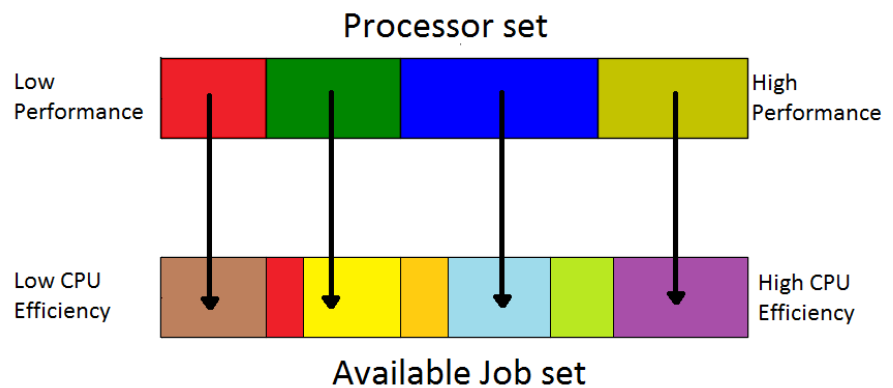

Figure 4: Scheduling scheme in the WLCG simulation: processor from the available processor sets are mapped to jobs in the available job set.

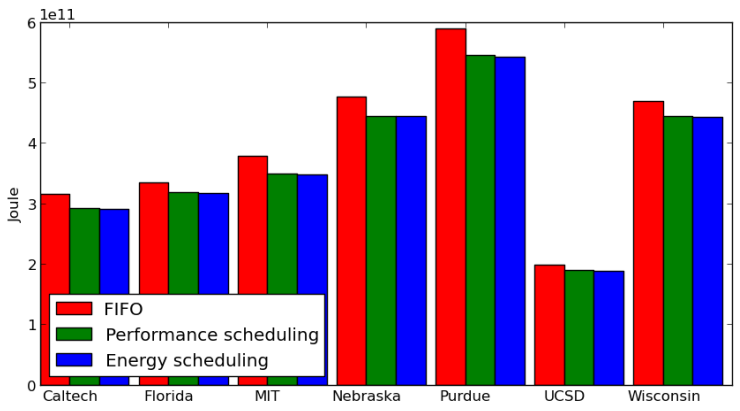

Figure 5: Energy consumption per site under three different scheduling algorithms.

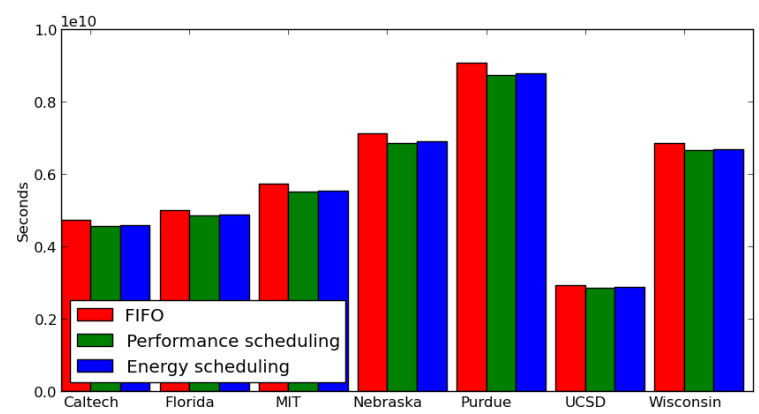

Figure 6: Performance per site under the three different algorithms.

These results don't identify energy or performance scheduling to be best, but clearly show that they should be preferred to job and processor agnostic schedulers. Given the focus in Tier2 sites is on performance the CPU performance scheduler will be in most cases the optimal choice.

\section{Conclusions and Future Work}

In the preceding sections, we have described general ideas as to how power optimizations could be made on a large distributed computing system like the WLCG. We take into account current and future computing models and foreseen technology evolution. 
The first step we are taking is to model distributed systems such as the WLCG that present different power cost functions, heterogeneous hardware choices and serve a diverse range of applications. Our upcoming work will proceed in steps toward this goal. First we will determine what it is possible to measure today at each of the sites in terms of power use, as well as possible variations the power costs in WLCG computer centers. Secondly, this macro knowledge will be coupled with further characterization of the evolving power and performance characteristics of available hardware, including coprocessors and SoCs. Ultimately by determining what node/processor level power savings might be possible through application choices, we will be able to develop power aware applications on heterogeneous hardware and we will examine power and throughput impact of power-aware scheduling.

In this paper we have discussed the application and exploitation of various techniques to reduce power consumption and cost in distributed high throughput computing clusters such as the WLCG. Such techniques will be of use to build the computing systems of ever greater computational throughput required for the scientific problems of the next decade and beyond.

\section{Acknowledgments}

This work was partially supported by the National Science Foundation, under Cooperative Agreement PHY-1120138, and by the U.S. Department of Energy.

\section{References}

[1] Bird I 2011 Computing for the Large Hadron Collider Annual Review of Nuclear and Particle Science 61 99-118

[2] Evans L and Bryant P 2008 LHC Machine JINST 3 S08001

[3] Chatrchyan $\mathrm{S}$ et al (CMS Collaboration) 2012 Observation of a new boson at a mass of $125 \mathrm{GeV}$ with the CMS experiment at the LHC Phys.Lett. B716 30-61

[4] Aad G et al (Atlas Collaboration) 2012 Observation of a new particle in the search for the Standard Model Higgs boson with the ATLAS detector at the LHC Phys.Lett. B716 1-29

[5] Chatrchyan S et al (CMS Collaboration) 2008 The CMS experiment at the CERN LHC JINST 3 S08004

[6] Aad G et al (Atlas Collaboration) 2008 The Atlas Experiment at the CERN Large Hadron Collider JINST 3 S08003

[7] http://www.top500.org/

[8] Rossi L and Bruning O 2012 High Luminosity Large Hadron Collider - A description for the European Strategy Preparatory Group, CERN report CERN-ATS-2012-236

[9] Bayatyan G L et al 2005 CMS Computing Technical Design Report, CERN Report CERN-LHCC-2005-023

[10] Rehn J et al 2006 PhEDEx high-throughput data transfer management system Proceedings of Computing in High Energy Physics (CHEP 2006), Mumbai

[11] Fuller S H and Millet L I (Editors) 2011 The Future of Computing Performance: Game Over or Next Level? The National Academies Press. 
[12] Jones C D, Elmer P, Sexton-Kennedy L, Green C and Baldooci A 2011 Multi-core aware applications in CMS J. Phys.: Conf. Ser. 331042012

[13] Jones C D and Sexton-Kennedy E 2014 Stitched Together: Transitioning CMS to a Hierarchical Threaded Framework, J. Phys. Conf. Ser. 513022034

[14] Clemencic M, Hegner B, Mato P and Piparo D 2014 Introducing Concurrency in the Gaudi Data Processing Framework, J. Phys. Conf. Ser. 513022013

[15] Ekman M, Warg F and Nilsson J 2005 An In-depth Look at Computer Performance Growth SIGARCH Comput. Archit. News 33 144-147

[16] CERN IT Technology, Market and Cost Trends 2012, available at http://wlcg.web.cern.ch/news/technology-market-cost-trends

[17] Elmer P, Rappoccio S, Stenson K and Wittich P 2013 The Need for an R\&D and Upgrade Program for CMS Software and Computing [arXiv:1308.1247]

[18] Mattmann J et al 2012 Track finding in ATLAS using GPUs J. Phys. Conf. Ser. 396022035

[19] Halyo V, LeGresley P, Lujan P, Karpusenko V and Vladimirov A 2014 First Evaluation of the CPU, GPGPU and MIC Architectures for Real Time Particle Tracking based on Hough Transform at the LHC, JINST 9 P04005

[20] Funke D, Hauth T, Innocente V, Quast G, Sanders P and Schieferdecker D 2014 Parallel track reconstruction in CMS using the cellular automaton approach, J. Phys. Conf. Ser. 513052010

[21] Amerio S et al Many-core applications to online track reconstruction in HEP experiments, J. Phys. Conf. Ser. $\mathbf{5 1 3} 012002$

[22] Amerio S et al 2013 Applications of Many-Core Technologies to On-line Event Reconstruction in High Energy Physics Experiments, presented at IEEE NSS 2013.

[23] Clark P J et al. 2011 Algorithm acceleration from GPGPUs for the ATLAS upgrade J. Phys. Conf. Ser. 331022031

[24] Emeliyanov D and Howard J 2012 GPU-based tracking algorithms for the ATLAS high-level trigger J. Phys. Conf. Ser. 396012018

[25] Hauth T, Innocente V and Piparo D 2012 Development and Evaluation of Vectorised and Multi-Core Event Reconstruction Algorithms within the CMS Software Framework J. Phys. Conf. Ser. 396 052065

[26] Andreassen R, Meadows B T, de Silva M, Sokoloff M D and Tomko K 2014 GooFit: A library for massively parallelising maximum-likelihood fits, J. Phys. Conf. Ser. 513052003

[27] Gorbunov S et al 2011 ALICE HLT High Speed Tracking on GPU IEEE Trans. Nucl. Sci. 58

[28] Hagiwara K, Kanzaki J, Li Q, Okamura N, and Stelzer T 2013 Fast computation of MadGraph amplitudes on graphics processing unit (GPU), submitted to Eur. Phys. J. C

[29] Merck M et al. 2012 IceCube's GPGPU cluster for extensive MC production J. Phys. Conf. Ser. 396 022046

[30] Abdurachmanov D, Elmer P, Eulisse G and Muzaffar S 2014 Initial Explorations of ARM Processors for Scientific Computing, J. Phys. Conf. Ser. 523 012009 [arXiv 1311.0269]

[31] Abdurachmanov D, Arya K, Bendavid J, Boccali T, Cooperman G, Dotti A, Elmer P, Eulisse G, Giacomini F, Jones C D, Manzali M and Muzaffar S 2014 Explorations of the Viability of ARM and Xeon Phi for Physics Processing, J. Phys. Conf. Ser. 513052008 [arXiv 1311.1001] 
[32] Clemencic M, Couturier B, Neufeld N and Subbiah V K 2014 Measurements of the LHCb software stack on the ARM architecture, J. Phys. Conf. Ser. 513052014

[33] Rajovic N, Carpenter P M, Gelado I, Puzovic N, Ramirez A and Valero M 2013 Supercomputing with commodity CPUs: are mobile SoCs ready for HPC?, Proceedings of SC13: International Conference for High Performance Computing, Networking, Storage and Analysis (SC13), Article 40.

[34] Bloom K et al (CMS Collaboration) 2014 CMS Use of a Data Federation, J. Phys. Conf. Ser. 513 042005

[35] Dorigo A, Elmer P, Furano F and Hanushevsky A 2005 XROOTD - A highly scalable architecture for data access WSEAS Transactions on Computers 4.3 (2005)

[36] Furano F, Elmer P, Hanushevsky A and Gerardo G 2006 Latencies and Data Access. Boosting the performance of distributed applications Proceedings of Computing in High Energy Physics (CHEP 2006), Mumbai

[37] Barroso L and Holzle U 2007 The Case for Energy-Proportional Computing IEEE Computer 40

[38] Qureshi A, Weber R, Balakrishnan H, Guttag J, Maggs B 2009 Cutting the electric bill for internet-scale systems, ACM SIGCOMM Comput. Commun. Rev 39(4), 123-134

[39] Rao L, Liu X, Xie L, Liu W 2010 Minimizing electricity cost: optimization of distributed internet data centers in a multi-electricity-market environment, in INFOCOM, 2010 Proceedings IEEE. IEEE, San Diego, pp 1-9

[40] M. Makkes, A. Taal, A. Osseyran and P. Grosso A decision framework for placement of applications in clouds that minimizes their carbon footprint In: Journal of Cloud Computing: Advances, Systems and Applications 2013, Vol.2

[41] Q. Chen , P. Grosso , K. van der Veldt , C. de Laat, R. Hofman , H. Bal, Profiling Energy Consumption of VMs for Green Cloud Computing, Proceedings of the 2011 IEEE Ninth International Conference on Dependable, Autonomic and Secure Computing, p.768-775, December 12-14, 2011

[42] X. Ma, M. Dong, L. Zhong, and Z. Deng, "Statistical power consumption analysis and modeling for gpu-based computing", SOSP Workshop on Power Aware Computing and Systems (HotPower09), 2009.

[43] H. Nagasaka, N. Maruyama, A. Nukada, T. Endo, and S. Matsuoka, "Statistical power modeling of GPU kernels using performance counters", The first international green computing conference, 2010.

[44] J. Chabarek, J. Sommers, P. Barford, C. Estan, D. Tsiang, and S. Wright, "Power Awareness in Network Design and Routing”, INFOCOM, 2008

[45] Future Generation Computer Systems Special Section: Energy efficiency in large-scale distributed systems Volume 28, Issue 5, Pages 709-822 (May 2012) Edited by Tuan Anh Trinh, Helmut Hlavacs and Domenico Talia

[46] The Scientific Grand Challenges: Architectures and Technology for Extreme Scale Computing, December 8-10, 2009, San Diego, CA (DOE ASCR 2011)

[47] Exascale Programming Challenges: Report of the 2011 Workshop on Exascale Programming Challenges, July 27-29, 2011, Marina del Rey, CA (DOE ASCR 2011)

[48] Ruben Janssen - "Reducing the energy consumption and costs of the WLCG by predicting job CPU efficiency", unpublished Bachelor Thesis 2014 UvA (supervisors: dr. P. Grosso and dr. P. Elmer)

[49] Andreeva J et al 2012 Experiment Dashboard - a generic, scalable solution for monitoring of the LHC computing activities, distributed sites and services J. Phys. Conf. Ser. 396032093 